\title{
Effect of Water on Nano-rheology of Dioctylphthalate Confined between Surfaces Coated with Long Alkyl Chains
}

\author{
Yoshisada Kayano ${ }^{1,2}$, Hiroshi Sakuma ${ }^{1}$ and Kazue Kurihara ${ }^{1, *}$ \\ ${ }^{1}$ Institute of Multidisciplinary Research for Advanced Materials, Tohoku University, Katahira, Aoba-ku, Sendai 980-8577, Japan. \\ ${ }^{2}$ Shiraishi kogyo kaisha, Itd., 78, 4, Motohama-cho, Amagasaki, 660-0085, Japan. \\ * Fax: 81-22-217-5674, e-mail: kurihara@tagen.tohoku.ac.jp
}

To study rheological properties of precipitated calcium carbonate (PCC) dispersion in dioctylphthalate (DOP) in the presence of a small amount of water, ca. $770-2600 \mathrm{ppm}$, the macroscopic viscosity was measured using a B-type viscometer. The PCC coated with stearic acid dispersed in DOP exhibited higher viscosity than the uncoated PCC. To know the mechanism of this high viscosity, the rheological properties of DOP in nanometer space were investigated using the shear resonance measurement. For this measurement, we used mica surfaces modified with dioctadecyldimethylammoniumbromide (DODA) as a model of PCC surfaces which bears long alkyl chains, and the effects of the surface modification and the addition of water to DOP were examined. For the $1164 \mathrm{ppm}$ water content, we observed that the viscosity of DOP between DODA modified surfaces started to increase at the distance of $57.1 \mathrm{~nm}$, which is much longer than the value for bare mica surfaces, $10.7 \mathrm{~nm}$. When the water content was decreased to $469 \mathrm{ppm}$, the viscosity started to increase at small distances, $1.1 \mathrm{~nm}$ and $8.0 \mathrm{~nm}$ for the DODA modified and bare mica surfaces, respectively. These distances were compared with the average particle distance in a DOP sol employed for the macroscopic rheological measurement. Results of the current study indicated that a mechanism of the high viscosity of dispersion of PCC coated with long alkyl chains in DOP would be due to the increasing viscosity of DOP by the combined effects of water and long alkyl chains of the PCC surfaces.

Key words: DOP, PCC, viscosity, shear resonance measurement, long alkyl chain, water content

\section{INTRODUCTION}

Colloidal particles are widely used as materials in industrial products ${ }^{1,2}$ as well as those in nano-technology ${ }^{3}$. It is important to regulate the dispersive and rheological properties of colloidal particles for many applications. The use of surfactants for surface modifications is effective for regulating these properties ${ }^{4,5}$. Colloidal nano-particles of precipitated calcium carbonate (PCC) coated with stearic acid and other fatty acids have been increasingly used as a rheological modifier with the plasticizer of dioctylphthalate (DOP) for paints and sealants ${ }^{6,7}$. We recently found that the high viscosity of the PCC dispersion in DOP (DOP sol) was due to the increasing viscosity of DOP confined in nanometer thickness between surfaces coated with long alkyl chains. We attributed the high viscosity to the interdigitation of alkyl chains among DOP molecules and DOP and surface DODA molecules ${ }^{8}$. Another important factor in the regulation of the rheological properties of the solid dispersions is the water content ${ }^{9}$. Therefore it is important to know the effect of water on the rheological properties of DOP confined between the surfaces coated with long alkyl chains.

Here, we studied the effect of water on the viscosity of DOP sol by macroscopic approach using a viscometer and nano-scopic approach using the shear resonance measurement. The shear measurement methods employing the surface forces apparatus (SFA) have been developed for investigating confined liquids in a nano-space ${ }^{10-14}$. The resonance mode ${ }^{13-18}$ developed by us has a high sensitivity for investigating the viscosity, friction and lubrication of confined liquids between solid surfaces, and is easy to use because we monitor relatively large resonance signals.

\section{EXPERIMENTAL SECTION}

2.1 Measurements of macroscopic viscosity of DOP sol

We used commercially available $P C C$ coated with stearic acid (SHIRAISHI KOGYO KAISHA, Ltd.) and uncoated PCC (SHIRAISHI KOGYO KAISHA, Ltd.). The particles of coated and uncoated PCC were rhombohedral and uniform in size (ca. $80 \mathrm{~nm}$ in side). Industrial DOP ( $99 \%$ purity, New Japan Chemical Co., Ltd., Fig. 1a) was used to prepare the DOP sol.

(a)

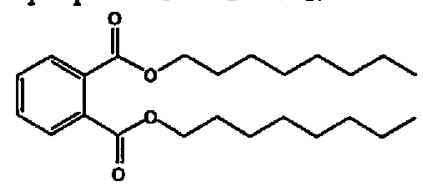

(b)

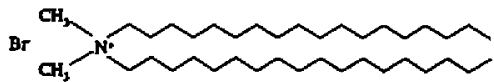

Fig.1. Molecular structures of (a) DOP and (b) DODA. 
DOP (400 g) and PCC (300 g) were thoroughly mixed by the mixer (5DMV-01-r, DALTON Corp.). To measure the viscosity of the DOP sol, a B-type viscometer (TOKIMEC INC.) was used at a velocity of rotation of $20 \mathrm{rpm}$ during 3 minutes, and the temperature was $20.0 \pm 0.1{ }^{\circ} \mathrm{C}$. The water contents of DOP sol were measured by the Karl-Fischer titration instrument (HIRANUMA AQUACOUNTER AQ-7).

\subsection{Shear resonance measurement}

DOP (di-n-octylphthalate, WAKO Chemicals, standard grade, $>99.9 \%$ in purity) was used for the shear resonance measurements. The water contents of DOP were measured by the Karl-Fischer titration instrument. To investigate the effect of water on the viscosity of DOP sol, DOP of high (1164 ppm) and low (469 ppm) water contents were prepared. The mica sheets (1 $\times 1 \mathrm{~cm}^{2}$, thickness $\left.1-3 \mu \mathrm{m}\right)$ as the substrates were freshly cleaved, and the silver was deposited on one side of them at a thickness of $50 \mathrm{~nm}$ using a standard procedure ${ }^{18}$. The sheets were then glued onto cylindrical silica disks (radius of curvature, $20 \mathrm{~mm}$; diameter of the disc; $10 \mathrm{~mm}$ ) with the silver-coated sides down on the disk, and subjected to the experiment. Here we used the dioctadecyldimethylammoniumbromide (DODA) (Sogo Pharmaceutical Co., Fig. 1b) was deposited on the mica surface using the Langmuir-Blodgett (LB) method (USI-2-00KC, USI Co., Ltd.) ${ }^{19}$.

The main part of the shear resonance apparatus was shown in Fig. 2. The distance between the surfaces $(D)$ was determined with $0.1 \mathrm{~nm}$ in resolution using the fringes of equal chromatic order (FECO). The shear was applied to the DOP by the lateral oscillation of the upper surface using a four-sectored piezo tube, which was driven by a sinusoidal input voltage, $U_{\mathrm{in}}$. The amplitude of shear response was obtained as the output voltage $U_{\text {out }}$ using the capacitance probe.

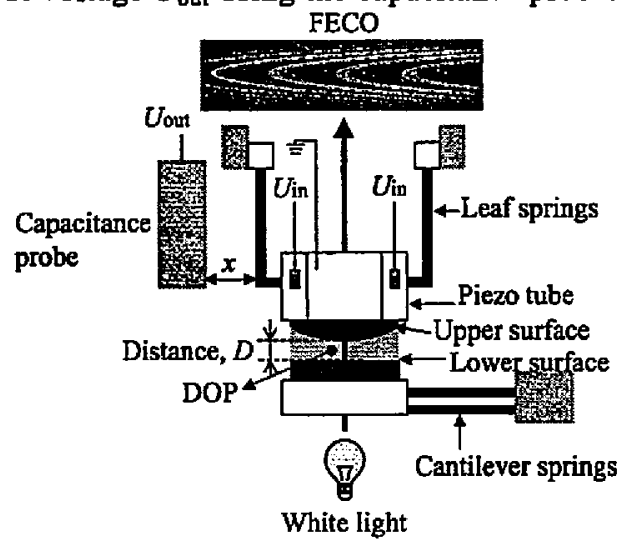

Fig.2. Main part of shear resonance apparatus.

The control measurements were carried out with the surfaces separated and contacted in air. The resonance frequency of the surfaces separated $\left(\Omega_{0, \mathrm{SP}}\right)$ in air is determined by the mass and the spring constant of the upper surface unit. While the resonance frequency of the surfaces contacted $\left(\Omega_{0, \mathrm{MC}}\right)$ in air is determined by the combination of the mass and spring constant of both the upper and lower surface units. After the control measurements, DOP $(10 \mu \mathrm{L})$ was injected into the gap between the surfaces. The measurements were started at $D=200 \sim 400 \mathrm{~nm}$ and the resonance curves were recorded at various separations on approach. The temperature was kept at $23.8 \pm 0.1^{\circ} \mathrm{C}$.

\section{RESULTS AND DISCUSSION}

3.1 Viscosity of DOP sol studied by a viscometer

Macroscopic viscosities of DOP sol of coated and uncoated PCC as a function of water content were measured using a B-type viscometer as shown in Fig. 3. The viscosities of DOP sol of coated PCC were gradually increased with the increasing water content from 922 to $1377 \mathrm{ppm}$ and steeply increased with the increasing water content from 1377 to $2175 \mathrm{ppm}$. On the other hand, for uncoated PCC, the viscosity of DOP sol was low and showed no significant difference in the water concentration range from 770 to 2121 ppm. It started sharply to increase with the increasing water content beyond $2121 \mathrm{ppm}$. This effect of water is more pronounced in the DOP sol of coated PCC than that of uncoated PCC.

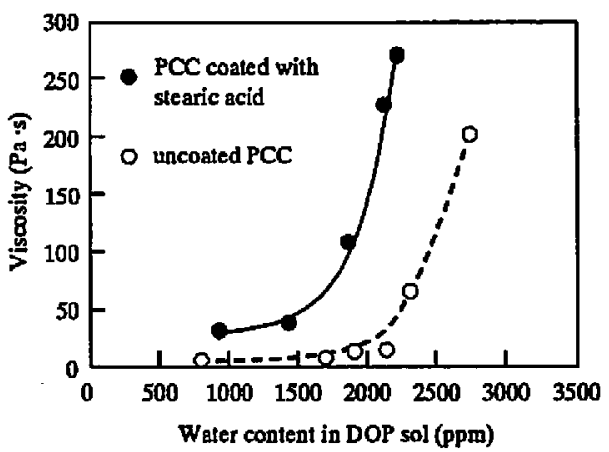

Fig. 3. Viscosity of DOP sols of PCC coated with stearic acid and uncoated against water contents of DOP sols.

In order to elucidate the mechanism of the high viscosity of DOP sol with high water contents, we investigated the rheological properties of DOP confined in a nano-space.

3.2 High water content DOP between the DODA modified mica surfaces

The resonance curves for the DOP at higher water content $(1164 \mathrm{ppm}), \mathrm{DOP}_{\mathrm{HW}}$, confined between the DODA modified mica surfaces were shown in Fig. 4. The resonance peak at $D=320.6$ $\mathrm{nm}$ appeared at the angular frequency of $209 \mathrm{~s}^{-1}$ that was same as the $\Omega_{0, \mathrm{SP}}\left(209 \mathrm{~s}^{-1}\right)$. The peak amplitude decreased from the amplitude in air, reflecting the higher viscosity of $\mathrm{DOP}_{\mathrm{HW}}$ than that of air. The amplitude of the resonance peak gradually decreased with decreasing $D$ from $320.6 \mathrm{~nm}$ to $63.8 \mathrm{~nm}$. This indicated that the viscosity of the DOP $_{\mathrm{HW}}$ gradually increased with the decreasing distance. At $D=57.1 \mathrm{~nm}$, the resonance peak abruptly disappeared and a broad 
curve (resonance frequency, $311 \mathrm{~s}^{-1}$ ) emerged. These results mean that the viscosity of $D O P_{H W}$ started to sharply increase at this distance and transferred the movement of the upper surface to the lower one leading the two surfaces couple. When $D$ was further decreased, the resonance peak shifted toward the $\Omega_{0, M C}\left(422 \mathrm{~s}^{-1}\right)$, while the intensity remained low. At $D=9.9 \mathrm{~nm}$, the peak appeared at a frequency close to $\Omega_{0, \mathrm{MC}}$, though the amplitude of the peak was still low compared with the reference state, the DODA modified mica surfaces in contact in air.

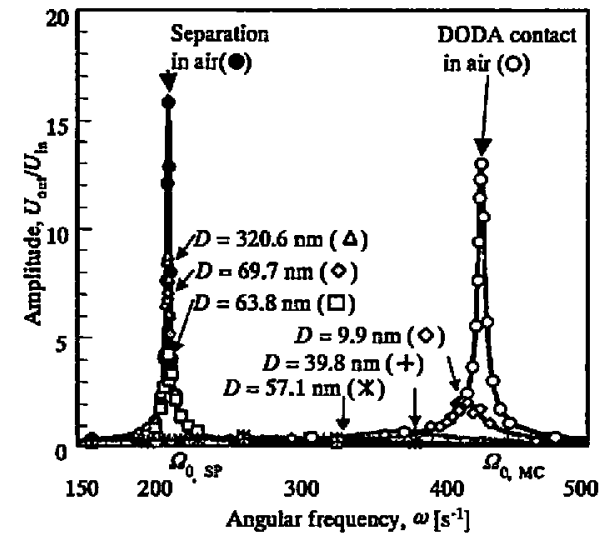

Fig. 4. Resonance curves of the DOP ${ }_{H w}$ confined between the DODA modified surfaces at various separation distances, $D$.

3.3 Low water content DOP between DODA modified mica surfaces

The resonance curves of the DOP at lower water content (469 ppm), DOP $\mathrm{LW}_{\mathrm{LW}}$ confined between the DODA modified mica surfaces are shown in Fig. 5. At $D=249.6 \mathrm{~nm}$, the resonance peak appeared at the angular frequency of the $\Omega_{0, S P}\left(208 \mathrm{~s}^{-1}\right)$. The amplitude of the resonance peaks gradually decreased with the decreasing $D$ from $249.6 \mathrm{~nm}$ to $4.6 \mathrm{~nm}$. At $D=1.1 \mathrm{~nm}$, the resonance peak suddenly disappeared and a broad curve emerged. This means that the viscosity of DOP $_{\text {Lw }}$ started to sharply increase at this surface separation.

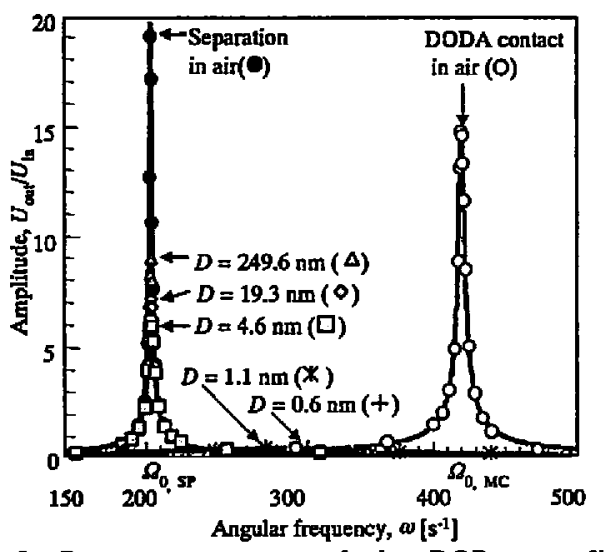

Fig. 5. Resonance curves of the $D O P_{L w}$ confined between the DODA modified surfaces.

The distance where the resonance peaks disappeared upon approach was $57.1 \mathrm{~nm}$ for the
DOP $_{H W}$ confined between the DODA modified surfaces. This distance was much longer than that for the $\mathrm{DOP}_{1 \mathrm{w}}(1.1 \mathrm{~nm})$. This indicates that high water content of DOP indeed contributed to the increasing viscosity of the DOP confined between the surfaces with long alkyl chains at a long distance.

\subsection{Behavior of $\mathrm{DOP}_{\mathrm{HW}}$ and $\mathrm{DOP}_{\mathrm{LW}}$ between bare} mica surfaces

The resonance curves of the DOP ${ }_{\mathrm{HW}}$ confined between the bare mica surfaces are shown in Fig. 6. The resonance curves of the DOP $_{L w}$ between bare mica surfaces showed similar changes with those of the DOP $\mathrm{HW}$. The distances where the resonance peaks disappeared upon approach were $10.7 \mathrm{~nm}$ for the DOP $_{\mathrm{HW}}$ and $8.0 \mathrm{~nm}$ for the DOP $_{L w}$ between bare mica surfaces. For bare mica surface, the distances where the viscosity started to sharply increase were not influenced by water and much smaller than $57.1 \mathrm{~nm}$ for the DOP $_{\text {HW }}$ between the DODA modified surfaces. The difference of the rhelogical properties of DOP between modified DODA and bare mica surfaces should be attributable to the presence of long alkyl chains. Therefore, the long alkyl chains on the surface as well as water in DOP are needed to increase the viscosity of DOP.

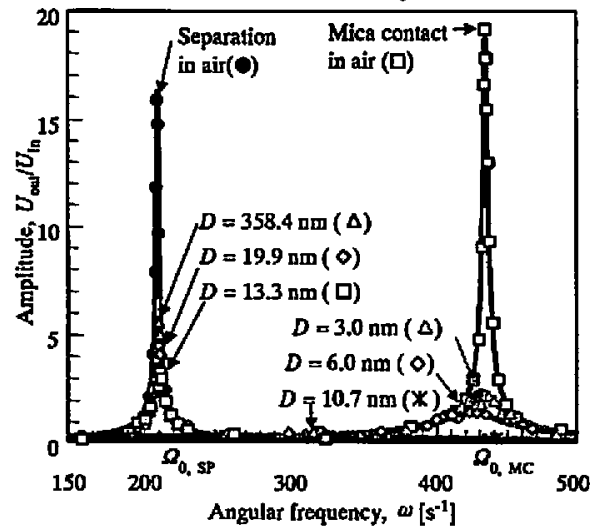

Fig. 6. Resonance curves of the $D P_{H w}$ confined between the bare mica surfaces.

3.5 Macro and nano-scopic viscosity of DOP sol

When the coated PCC was completely dispersed in the DOP sol, the average distance between the PCC particles was $51.4 \mathrm{~nm}$ which was quite consistent with the distance $(57.1 \mathrm{~nm})$ at which the viscosity of the $\mathrm{DOP}_{\mathrm{HW}}$ between the DODA modified mica surfaces started to sharply increase. This indicates that the high viscosity of DOP causes the high viscosity of DOP sol. One may note that for conditions (unmodified surfaces and low water contents) at which no large macroscopic viscosity was observed, the distance at which the viscosity of DOP started to sharply increase was much shorter, for example, $1.1 \mathrm{~nm}$ for $D O P_{L w}$ between DODA modified surfaces and ca. $10 \mathrm{~nm}$ for DOP between bare mica surfaces. Theses distances were much shorter than the average distance between PCC particles in the 
DOP sol. These results indicate that the low viscosities of DOP sol were due to the low viscosity of DOP. Consequently, we found that the combined effect of high water content (1164 ppm) and long alkyl chains of the surface increased the viscosity of DOP and this was the mechanism of high viscosity of DOP sol.

3.6 The plausible structure of DOP containing water on the surfaces modified with long alkyl chains

Here we discuss the effect of water on the structure of DOP molecules. In the condition we used for this study, the ratio of water to DOP was small such as DOP : water $=49: 1$ for the water content of $1164 \mathrm{ppm}$. First we will discuss the effect of the surface modification. It is known that the surface modified with long alkyl chains could orient liquid crystal molecules perpendicular to the surface ${ }^{20}$. Therefore, it should be reasonable to expect that DOP molecules bearing two $C_{B}$ long alkyl chains can be interdigitated in an array of long alkyl chains on the surface. Other DOP molecules are connected in the normal direction to the surfaces by forming the net-work employing the interdigitation of alkyl chains of DOP molecules as shown in Figure 7 . It is more difficult to interpret the effect of water, and we would like to propose two plausible effects. The first is that the oriented net-work of DOP molecules could be enhanced by the hydrogen bonding mediated by water molecules as shown in the inset of Fig. 7, resulting in the high viscosity of DOP confined between the DODA surfaces. The second is the formation of a reversed micellar type net-work of DOP. In any case, it is interesting that the organized structure of DOP extended in such a long distance of $57.1 \mathrm{~nm}$.

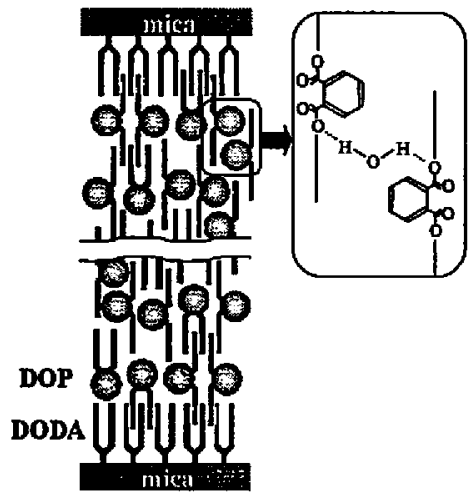

Fig. 7. Schematic drawings of one plausible structure of DOP (water content of $1164 \mathrm{pPm}$ ) confined between DODA modified surfaces.

\section{CONCLUSIONS}

We measured the macroscopic viscosity of DOP sol using a B-type viscometer. We also studied the rhological properties of the DOP of high (1164 ppm) and low (469 ppm) water contents confined between the DODA modified surfaces and the bare mica surfaces using the shear resonance measurement. We observed the large increase of the viscosity of DOP sol with the increasing water content. This can be explained by the high viscosity of DOP confined in a nano-space due to the combined effects of water and long alkyl chains of the PCC surface.

\section{ACKNOWLEDGEMENT}

This work was supported by the CREST program of Japan Science and Technology Agency (JST), and by a Grant-in-Aid for 21st Century COE Research, Giant Molecules and Complex Systems, from the Ministry of Education, Culture, Sports, Science and Technology of Japan.

\section{REFERENCES}

[1] H. Mouri and K. Akutagawa, Rubber Chem. Technol., 72, 960-968 (1999).

[2] M. Ambrosi, L. Dei, R. Giorgi, C. Neto, P. Baglioni, Langmuir, 17, 4251-4255 (2001).

[3] F. Caruso, R. A. Caruso, H. Mohwald, Science, 282, 1111-1114 (1998).

[4] M. Ettlinger, T. Ladwig, A. Weise, Prog. Org. Coat., 40, 31-34 (2000).

[5] A. A. Zaman, P. Singh, B. M. Moudgil, J. Colloid Interface Sci., 251, 381-387 (2002).

[6] R. M. Evans, "Polyurethane Sealants, Technology and Applications", Ed. by Technomic Publishing Company, Inc., Pennsylvania, (1993).

[7] Y. Kayano, I. Morioka, K. Hosoi, Material for imparting US2005-0004266-A1, 2005.

[8] Y. Kayano, H. Sakuma, K. Kurihara, Langmuir (2006) submitted.

[9] K. Cavalier, F. Larche, Colloid Surf. A-Physicochem. Eng. Asp., 197, 173-181 (2002).

[10] J. N. Israelachvili, P. M. McGuiggan, A. M. Homola, Science, 240, 189-191 (1988).

[11] J. Van Alsten, S. Granick, Phys. Rev. Lett., 61, 2570-2573 (1988).

[12] J. Klein, D. Perahia, S. Warburg, Nature, 352, 143-145 (1991).

[13] C. D. Dushkin, K. Kurihara, Colloid Surf. A-Physicochem. Eng. Asp., 130, 131-139 (1997).

[14] C. D. Dushkin, K. Kurihara, Rev. Sci. Instrum., 69, 2095-2104 (1998).

[15] K. Kurihara, Prog. Colloid Polym. Sci., 121, 49-56 (2002).

[16] M. Mizukami, K. Kusakabe, K. Kurihara, Prog. Colloid Polym. Sci., 128, 105-108 (2004).

[17] K. Mori, K. Kusakabe, T. Haraszti, K. Kurihara, Trans MRS-J, 26, 909-912 (2001).

[18] H. Sakuma, K. Otsuki, K. Kurihara, Phys. Rev. Lett., 96, 046104 (2006).

[19] K. Kurihara, K. Ohto, Y. Tanaka, Y. Aoyama, T. Kunitake, J. Am. Chem. Soc., 113, 444-450 (1991).

[20] J. Janik, R. Tadmor, J. Klein, Langmuir, 13, 4466-4473 (1997).

(Received December 9, 2006;Accepted April 17, 2007) 